\title{
HDAC8 and STAT3 repress BMF gene activity in colon cancer cells
}

\author{
Y Kang ${ }^{1}$, H Nian ${ }^{2}$, P Rajendran ${ }^{3}$ E Kim ${ }^{3}$, WM Dashwood ${ }^{3}$, JT Pinto ${ }^{4}$, LA Boardman ${ }^{5}$, SN Thibodeau ${ }^{5}$, PJ Limburg ${ }^{5}$, CV Löhr ${ }^{6}$, \\ WH Bisson ${ }^{7}$, DE Williams ${ }^{1,7}, \mathrm{E} \mathrm{Ho}^{1}$ and RH Dashwood ${ }^{\star, 3,8,9,10}$
}

\begin{abstract}
Histone deacetylase (HDAC) inhibitors are undergoing clinical trials as anticancer agents, but some exhibit resistance mechanisms linked to anti-apoptotic Bcl-2 functions, such as BH3-only protein silencing. HDAC inhibitors that reactivate BH3-only family members might offer an improved therapeutic approach. We show here that a novel seleno- $\alpha$-keto acid triggers global histone acetylation in human colon cancer cells and activates apoptosis in a p21-independent manner. Profiling of multiple survival factors identified a critical role for the BH3-only member Bcl-2-modifying factor (Bmf). On the corresponding BMF gene promoter, loss of HDAC8 was associated with signal transducer and activator of transcription 3 (STAT3)/specificity protein 3 (Sp3) transcription factor exchange and recruitment of p300. Treatment with a p300 inhibitor or transient overexpression of exogenous HDAC8 interfered with BMF induction, whereas RNAi-mediated silencing of STAT3 activated the target gene. This is the first report to identify a direct target gene of HDAC8 repression, namely, BMF. Interestingly, the repressive role of HDAC8 could be uncoupled from HDAC1 to trigger Bmf-mediated apoptosis. These findings have implications for the development of HDAC8-selective inhibitors as therapeutic agents, beyond the reported involvement of HDAC8 in childhood malignancy.
\end{abstract}

Cell Death and Disease (2014) 5, e1476; doi:10.1038/cddis.2014.422; published online 16 October 2014

Histone deacetylase (HDAC) enzymes have been implicated in both normal physiology and pathophysiology, and several HDAC inhibitors have entered clinical trials. ${ }^{1-3}$ These inhibitors include vorinostat, a direct-acting compound that fits the HDAC active site, and romidepsin, a prodrug that through reductive metabolism generates a zinc-binding thiol. ${ }^{4}$ Evaluation of such compounds against solid tumors and hematological malignancies has provided insights into clinical efficacy, as well as resistance mechanisms and off-target effects. $^{1-3}$

Off-target effects such as cardiotoxicity and thrombocytopenia arise from inhibitors acting on multiple HDACs, ${ }^{5,6}$ whereas resistance has been linked to anti-apoptotic $\mathrm{Bcl}-2$ functions, including $\mathrm{BH} 3-$ only protein silencing. ${ }^{7-9} \mathrm{HDAC}$ inhibitors that reactivate $\mathrm{BH}$-only family members, such as Bcl-2-modifying factor (Bmf), might offer an improved therapeutic approach for treating cancer and other conditions.

We previously reported on the HDAC inhibitory activity of methylselenopyruvate (MSP), a novel seleno- $a$-keto acid metabolite generated by transamination of the natural organoselenium compound methylselenocysteine (MSC). ${ }^{10-12}$ MSP was identified as a competitive inhibitor of HDAC8, and this was supported by molecular modeling studies of MSP/HDAC8 interactions in silico. ${ }^{10}$

HDAC8 was the first human HDAC to be crystalized with bound inhibitors, ${ }^{13-16}$ but it remains one of the least understood HDACs, with no known direct transcriptional target or corepressor partner. ${ }^{17,18}$ In pursuing the mechanism of action of MSP in human colon cancer cells, we identified a key role for HDAC8 in regulating BMF gene activity and Bmf-mediated apoptosis. The work provides support for HDAC8 as a relevant mechanistic target for cancer therapy, beyond its reported involvement in childhood cancer. ${ }^{19}$

\section{Results}

MSP triggers Bmf-mediated apoptosis independent of p21 induction. Metabolic conversion of MSC to MSP was reported previously. ${ }^{11}$ An inhibitor of aminotransfer reactions caused a dose-dependent loss of histone acetylation in human colon cancer cells treated with MSC, but not MSP (Figure 1a). This was associated with loss of cleaved poly(ADP-ribose)

\footnotetext{
${ }^{1}$ Linus Pauling Institute, Oregon State University, Corvallis, OR, USA; ${ }^{2}$ Department of Biostatistics, Vanderbilt University School of Medicine, Nashville, TN, USA; ${ }^{3}$ Center for Epigenetics \& Disease Prevention, Institute of Biosciences \& Technology, Texas A\&M Health Science Center, Houston, TX, USA; ${ }^{4}$ Department of Biochemistry \& Molecular Biology, New York Medical College, Valhalla, NY, USA; ${ }^{5}$ Mayo Clinic, Rochester, MN, USA; ${ }^{6}$ College of Veterinary Medicine, Oregon State University, Corvallis, OR, USA; ${ }^{7}$ Department of Environmental and Molecular Toxicology, Oregon State University, Corvallis, OR, USA; ${ }^{8}$ Department of Nutrition \& Food Science, Texas A\&M University, College Station, TX, USA; ${ }^{9}$ Department of Clinical Cancer Prevention, MD Anderson Cancer Center, Houston, TX, USA and ${ }^{10}$ Department of Molecular \& Cellular Medicine, Texas A\&M University College of Medicine, College Station, TX, USA

${ }^{*}$ Corresponding author: RH Dashwood, Center for Epigenetics \& Disease Prevention, Institute of Biosciences \& Technology, Texas A\&M Health Science Center, 2121 West Holcombe Boulevard, Houston, TX, USA. Tel/Fax: +1 7136777806; E-mail: rdashwood@ibt.tamhsc.edu

Abbreviations: Bmf/BMF, Bcl-2-modifying factor protein/gene; CdLS, Cornelia de Lange syndrome; ChIP, chromatin immunoprecipitation; HAT, histone acetyltransferase; HDAC, histone deacetylase; KMSB, $\beta$-keto-methylselenobutyrate; MSC, methylselenocysteine; MSP, methylselenopyruvate; MTT, 3-(4,5-dimethylthiazol-2-yl)-2,5diphenyltetrazolium bromide; NIPBL, nipped-B-like; STAT3, signal transducer and activator of transcription 3; PARP, poly(ADP-ribose)polymerase; RNA Pol II, RNA polymerase II; SAHA, suberoylanilide hydroxamic acid; SM, selenomethionine; Sp1, specificity protein 1; Sp3, specificity protein 3

Received 22.7.14; revised 27.8.14; accepted 02.9.14; Edited by M Diederich
} 


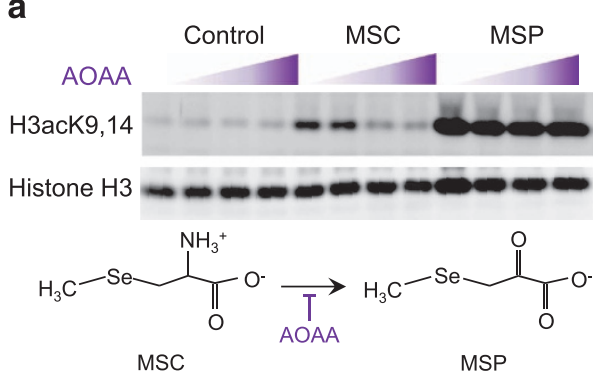

b

-++ AOAA - - + + AOAA

$-+{ }_{-}+\mathrm{MSC}-{ }_{-}-{ }_{-}+\mathrm{MSP}$

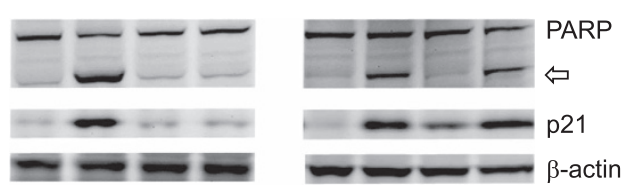

C

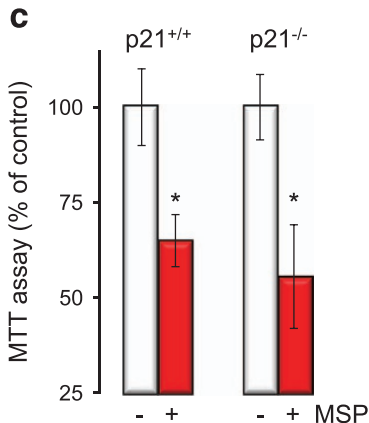

d

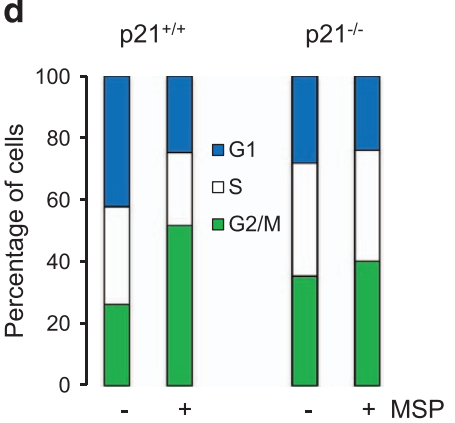

e

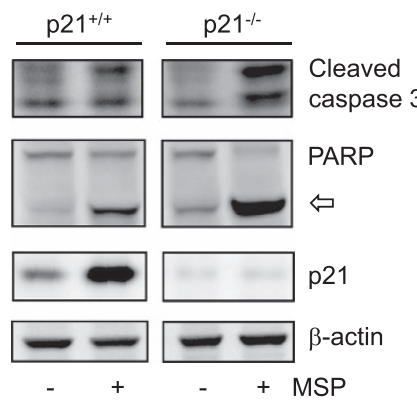

Figure 1 Induction of p21 by MSP is not a prerequisite for apoptosis in colon cancer cells. (a) HCT116 cells were incubated with the transaminase inhibitor aminooxyacetic acid (AOAA) $1 \mathrm{~h}$ before treatment with MSP, MSC, or vehicle (control). Whole-cell lysates were immunoblotted $6 \mathrm{~h}$ later for histone $\mathrm{H} 3$ acetylation. Wedge symbol indicates increasing concentration of AOAA. (b) AOAA pretreatment for $1 \mathrm{~h}$ blocked p21 induction by MSC and the cleavage of PARP, whereas MSP was unaffected by AOAA. Open arrow, cleaved PARP. (c) HCT116 $6^{\text {p21+/+ }}$ and HCT116 ${ }^{\text {p21-l- }}$ cells responded similarly to MSP treatment in MTT assays; mean \pm S.D., $n=3,{ }^{*} P<0.05$ using Students $t$-test. (d) $G_{2} / M$ cell cycle arrest at $48 \mathrm{~h}$ required the presence of p21. (e) In the absence of p21, MSP strongly increased cleaved caspase-3 and (open arrow) cleaved PARP. Results in a-e are representative findings from two or more independent experiments

polymerase (PARP) and p21 induction by MSC, whereas MSP was effective in the presence or absence of the transaminase inhibitor (Figure 1b). HCT116 ${ }^{\text {p21+/+ }}$ and HCT116 $6^{\text {p21-l- }}$ cells treated with MSP exhibited similar responses in the MTT (3-(4,5-dimethylthiazol-2-yl)-2,5-diphenyltetrazolium bromide) assay (Figure 1c), but $\mathrm{p}^{-1^{-/}}$cells did not accumulate in the $\mathrm{G}_{2} / \mathrm{M}$ phase of the cell cycle, unlike p $21^{+/+}$cells (Figure $1 \mathrm{~d}$ ). Notably, HCT116 ${ }^{\text {p21-/- }}$ cells had higher levels of cleaved caspase-3 and cleaved PARP compared with HCT116 $6^{\text {p21+/+ }}$ cells exposed to MSP under the same conditions (Figure 1e). These findings suggested that p21 induction is necessary for $\mathrm{G}_{2} / \mathrm{M}$ arrest, but is not a prerequisite for apoptosis induction by MSP.

To investigate the apoptotic mechanism in more detail, several survival factors were profiled. Induction of proapoptotic APAF1, BAK, BIM, and BMF, and loss of antiapoptotic $B C L X L$ occurred within $12 \mathrm{~h}$ of MSP treatment, in both HCT116 and HT29 colon cancer cell lines (Figure 2a). Induction of $B M F$ was particularly striking, and was confirmed both at the mRNA and protein level in time-course studies (Figures $2 \mathrm{~b}$ and $\mathrm{c}$ ). Knockdown of Bmf using RNAi attenuated the increase in BMF mRNA levels following MSP treatment (Figure 2d), and there was a reduction in Bmf protein expression (Figure 2e). These changes were associated with reduced PARP cleavage and attenuated levels of cleaved caspases (Figure 2f). Notably, in cells treated with MSP, activated caspases 3,6 , and 9 were reduced to near background levels by Bmf knockdown. Cleaved caspase-8 also was detected after MSP treatment and this caspase apparently was unaffected by Bmf knockdown (Figure 2f). Further experiments should seek to optimize Bmf knockdown beyond the $\sim 50-60 \%$ reduction achieved here (Figures $2 \mathrm{~d}$ and e), and clarify the relative contributions of internal and external apoptotic pathways. Nonetheless, we conclude that Bmf was a key mediator of MSP-induced apoptosis in colon cancer cells.

BMF activation by MSP involves HDAC8 and STAT3 de-recruitment. To prioritize histone marks for $B M F$ chromatin immunoprecipitation (ChIP) assays, immunoblotting was first performed on whole-cell lysates of colon cancer cells. At the early time point of $3 \mathrm{~h}, \mathrm{MSP}$ increased histone $\mathrm{H} 3$ acetylation (for example, H3acK9,14, H3acK9, and H3acK18) without dramatic changes in histone $\mathrm{H} 3$ methylation or phosphorylation (Supplementary Figure 1a). Histone $\mathrm{H} 4$ hyperacetylation also was detected within $3 \mathrm{~h}$ of MSP treatment, as evidenced by increased H4acK12 and H4acK5,8,12,16 histone marks (Supplementary Figure 1b). Time-course studies confirmed that histone hyperacetylation was maintained for several hours in response to MSP treatment, and that histone methylation marks associated with gene activation, such as $\mathrm{H} 3 \mathrm{~K} 4 \mathrm{me} 3$, became prominent after $24 \mathrm{~h}$ (Supplementary Figure 1c). These findings indicated that MSP induced global histone acetylation before alterations in other histone marks.

Because global histone acetylation changes preceded alterations in other histone modifications, we conducted ChIP assays in colon cancer cells at an early time point, $4 \mathrm{~h}$. Consistent with the BMF induction by MSP (Figure 2b), RNA polymerase II (Pol II) was recruited (Figure 3a) and there was a marked increase in local histone acetylation (Figure $3 b$ ). In parallel analyses of $B C L X L$ (Supplementary Figure 2), reduced histone acetylation and attenuated Pol II levels were 


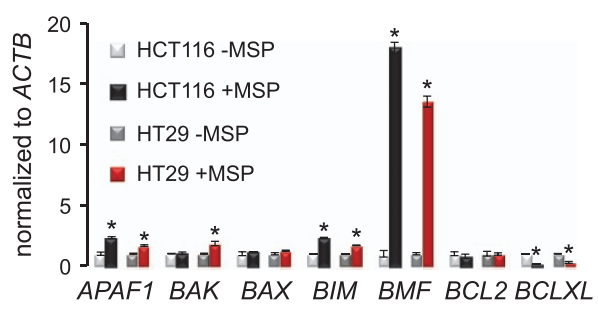

b

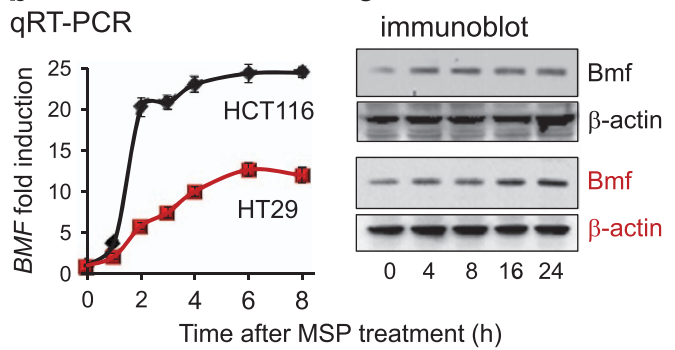

d

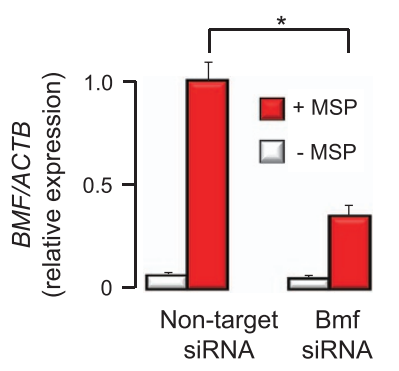

e

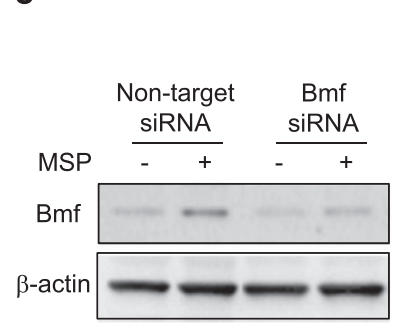

$\mathbf{f}$

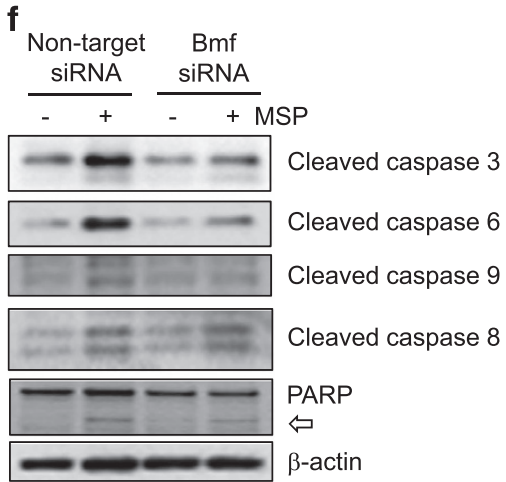

Figure 2 Bmf has a pivotal role in the apoptotic mechanism triggered by MSP. (a) Quantitative real-time PCR (qRT-PCR) assays of survival genes normalized to $\beta$-actin $(A C T B), 12 \mathrm{~h}$ after treatment of colon cancer cells with MSP $(10 \mu \mathrm{M})$ or vehicle. Data indicate mean \pm S.D., $n=3,{ }^{*} P<0.05$ using Students $t$-test to compare MSP-treated cells with vehicle controls. (b, $\mathbf{c})$ Time-course for induction of $B M F m R N A$ (mean \pm S.D., $n=3$ ) and Bmf protein expression. (d, e) In HCT116 cells, MSP was significantly less effective at inducing BMF mRNA (mean \pm S.D., $n=3,{ }^{*} P<0.05$ ) and Bmf protein expression following siRNA-mediated knockdown of Bmf. (f) Reduced cleavage of caspases and (open arrow) PARP in MSP-treated HCT116 cells following Bmf knockdown. Results in a-f are representative findings from two or more experiments

detected on the corresponding gene promoter after MSP treatment, consistent with the observed transcriptional downregulation of this pro-survival factor (Figure 2a). We did not examine serine 2-phosphorylated Pol II in association with $B C L X L$ or $B M F$, but this might be included in future experiments. ${ }^{20}$

The BMF promoter has predicted binding sites for multiple transcription factors, including Sp1 (specificity protein 1)/Sp3 (specificity protein 3) and signal transducer and activator of transcription 3 (STAT3). ChIP assays with the corresponding antibodies failed to identify consistent associations for Sp1, but upstream of the transcriptional start site there was binding of Sp3 and STAT3 (region 'f', Figures 3c and d). MSP treatment enhanced Sp3 and attenuated STAT3 interactions.

A prior report implicated HDAC1 as a repressor of BMF transcriptional activity. ${ }^{9}$ We confirmed HDAC1 interactions on the BMF promoter, slightly upstream of the transcription start site (peak region 'e', Figure $3 e$ ). The interactions of HDAC1 with BMF did not appear to be altered by MSP treatment. We also detected HDAC8 associations under constitutive conditions in colon cancer cells (open bars, peak region ' $h$ ', Figure $3 f)$. To our knowledge, $B M F$ is the first identified direct target gene for HDAC8 repression, none having been reported before. ${ }^{17}$ HDAC8 interactions were markedly reduced in response to MSP treatment (red bars, Figure $3 f$ ), and this coincided with increased histone acetyltransferase (HAT) p300 interactions (Figure $3 \mathrm{~g}$ ). Immunoblotting of whole-cell lysates showed no specific loss of HDAC8, or other class I HDACs, in response to MSP treatment (Supplementary Figure 3). We concluded that HDAC1 and HDAC8 might cooperate to maximally repress $B M F$, but HDAC8 de-recruitment alone was sufficient to activate the target gene.

Inhibition of p300 or overexpression of HDAC8 interferes with $B M F$ induction. On the basis of the ChIP data (Figures $3 f$ and g), HDAC8 and p300 were examined further as regulators of $B M F$ transcription. Treatment of colon cancer cells with a p300 inhibitor attenuated significantly the basal expression levels as well as the dose-dependent induction of $B M F$ by MSP (Figure 4a). In transient transfection experiments, forced expression of HDAC8 also interfered with the ability of MSP to induce the target gene (Figure $4 b$ ), supporting a reciprocal relationship between p300 and HDAC8 in regulating BMF transcriptional activity. Although the results in Figure $4 a$ were optimized for the specific p300 inhibitor selected, incomplete reductions in BMF expression were obtained, suggesting the possible involvement of other HATs in regulating BMF. Gain- and loss-of-function experiments, including the use of HAT mutants and HDAC8 silencing, might provide additional insights.

\section{HDAC8 and STAT3 cooperate to repress BMF transcription.} To clarify the apparent reciprocal relationship between Sp3 and STAT3 in regulating BMF (Figures $3 c$ and $d$ ), we performed RNAi experiments on the corresponding transcription factors. Knockdown of STAT3 increased the constitutive and MSP-induced levels of BMF, supporting a suppressive role for STAT3 on the BMF promoter (Figure 5a). Unexpectedly, knockdown of Sp3 did not produce a corresponding decrease in BMF expression, nor was there an apparent 

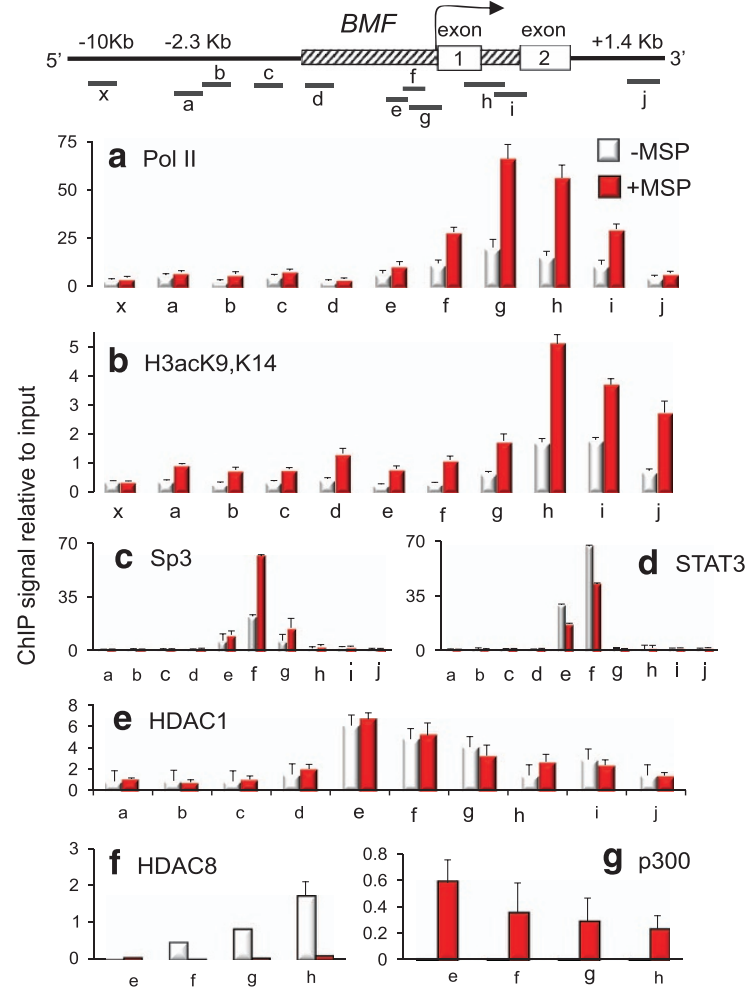

Figure 3 HDAC8 is a repressor of the BMF gene. Primers were designed to interrogate different regions of the BMF gene, including the promoter (hatched box) and flanking sequences. (a) MSP treatment enhanced RNA Pol II recruitment and (b) local histone acetylation levels. (c) MSP increased Sp3 transcription factor binding, adjacent to the transcription start site (arrow), and (d) reduced STAT3 associations. (e) HDAC1 interactions with the BMF promoter were unaffected by MSP treatment. (f) HDAC8 was present on BMF under constitutive conditions and was de-recruited following MSP treatment, coincident with increased p300 associations (g). ChIP assays were conducted at $4 \mathrm{~h}$ in HCT116 cells treated with MSP (+MSP) or vehicle (-MSP). Data were normalized to input controls; H3acK9,K14 also was normalized to histone $\mathrm{H} 3$. Results in $\mathbf{a}-\mathbf{g}$ indicate mean \pm S.D., $n=3$, from a single experiment that was repeated on three or more separate occasions a

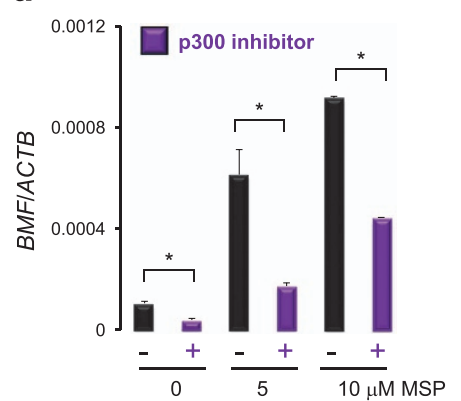

b
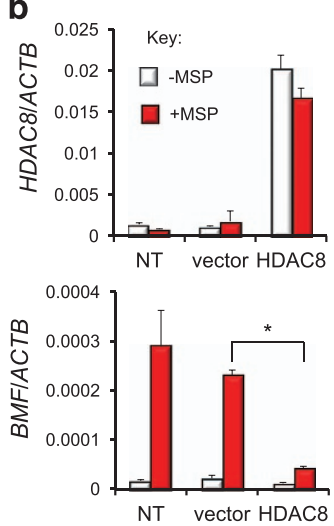

Figure 4 Inhibition of $\mathrm{p} 300$ and HDAC8 overexpression interferes with BMF transcription. (a) Pretreatment of colon cancer cells with p300 inhibitor $\mathrm{C}^{646^{46}}(10 \mu \mathrm{M})$ reduced the constitutive levels of $B M F$ and interfered with induction of the target gene by MSP. (b) Forced expression of HDAC8 by transient transfection rescues cells from BMF induction by MSP. In $\mathbf{a}, \mathbf{b}$, bars indicate mean \pm S.D., $n=3$, from a single experiment that was repeated at least twice $\left({ }^{*} P<0.05\right.$, by Student's $t$-test) compensatory mechanism involving Sp1 upregulation after Sp3 silencing (Figure 5a). These results suggested a possible 'bystander' effect in which Sp3 interacts with a cognate binding site that becomes unmasked following STAT3 de-recruitment, without necessarily activating the target gene. We conclude that in addition to STAT3 and Sp3, other factors regulate BMF transcriptional activity on HDAC8 release, possibly in association with HDAC1.

Finally, HDAC8 was immunoprecipitated from whole-cell lysates of HCT116 cells and immunoblotted for potential protein partners. No interaction with Sp3 was detected, but a band corresponding to STAT3 was identified (Figure 5b, open arrow). To our knowledge, this is the first evidence reported for a protein partner in the HDAC8 corepressor complex. ${ }^{17}$ Notably, the STAT3 band was detected in the presence and absence of MSP treatment (Figure $5 b$ ), suggesting that an HDAC8/STAT3 corepressor complex might be released from the $B M F$ promoter to recruit $\mathrm{p} 300$ and other coactivators (Figure 6).

\section{Discussion}

The first X-ray crystallography structures published of a human HDAC, HDAC8, ${ }^{13,14}$ provided valuable insights into the catalytic mechanism and binding characteristics of various inhibitors. A decade later, surprisingly little information was available on the role of HDAC8 in physiology or pathophysiology. HDAC8 associates with $a$-actin and regulates smooth muscle contractility, ${ }^{21}$ as well as influencing human myometrial activity via the acetylation of heat-shock protein $20 .^{22}$ Other class I HDACs (HDAC1, HDAC2, and HDAC3) are known to associate with gene promoters in repressor complexes, but promoter interactions with HDAC8 have not been reported, nor have regulatory gene targets of HDAC8 been clearly described. ${ }^{17}$ We now report, for the first time, that HDAC8 can associate with the BMF gene under constitutive conditions in colon cancer cells, and establish a pro-survival scenario via the repression of Bmf protein levels.

The increase in global histone acetylation in colon cancer cells treated with MSP was recapitulated in several other cancer cell lines. For example, time-course studies in $\mathrm{BE}(2) \mathrm{C}$ neuroblastoma cells revealed a rapid induction of histone acetylation by MSP compared with MSC parent compound (Supplementary Figure 4a). Interestingly, MSC, MSP, and the structural analog $\beta$-keto-methylselenobutyrate (KMSB) also induced global histone acetylation in prostate, breast, lung, and leukemia cells, whereas selenomethionine (SM) had no such effect (Supplementary Figure 4b). Unlike MSC, SM is a poor substrate for aminotransferase activity. ${ }^{12}$ In a pilot study in vivo, we observed that dietary MSC, but not SM, inhibited mouse colon tumor multiplicity, and this was associated with increased Bmf expression and histone acetylation in the tumors, without marked changes in overall HDAC8 protein levels (Supplementary Figure 5). We are now seeking to test the in vivo efficacy of MSP and KMSB along with the corresponding parent compounds as inducers of Bmf. Further insights also might come from ChIP assays following treatment with MSC and a transaminase inhibitor, such as aminooxyacetic acid (AOAA; Figure 1); however, such experiments would have to be optimized to account for the 


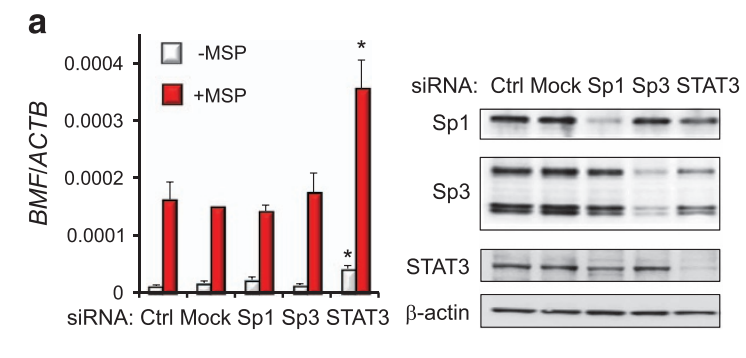

b

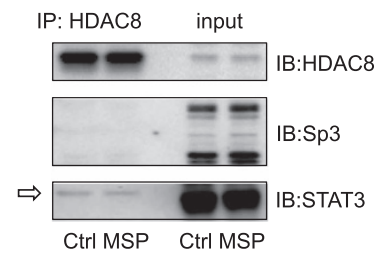

Figure 5 STAT3 associates with HDAC8 and represses BMF transcription. (a) siRNAs were used to target STAT3, Sp1, or Sp3, with transfection reagent (mock) and untreated controls (Ctrl) included in the experiment. After $48 \mathrm{~h}$ RNAi knockdown, cells were treated with MSP or vehicle for $12 \mathrm{~h}$ and BMF expression was determined relative to ACTB. Data $=$ mean \pm S.D., $n=3$, from a single experiment that was repeated twice $\left({ }^{*} P<0.05\right.$, by Student's $t$-test). Knockdown of STAT3, Sp1, and Sp3 was confirmed by immunoblotting (IB). (b) Immunoprecipitation (IP) with HDAC8 antibody followed by IB with the corresponding antibodies identified a band for STAT3 (open arrow) but not Sp3 or Sp1 (data not shown). Results are representative of the findings from three independent experiments

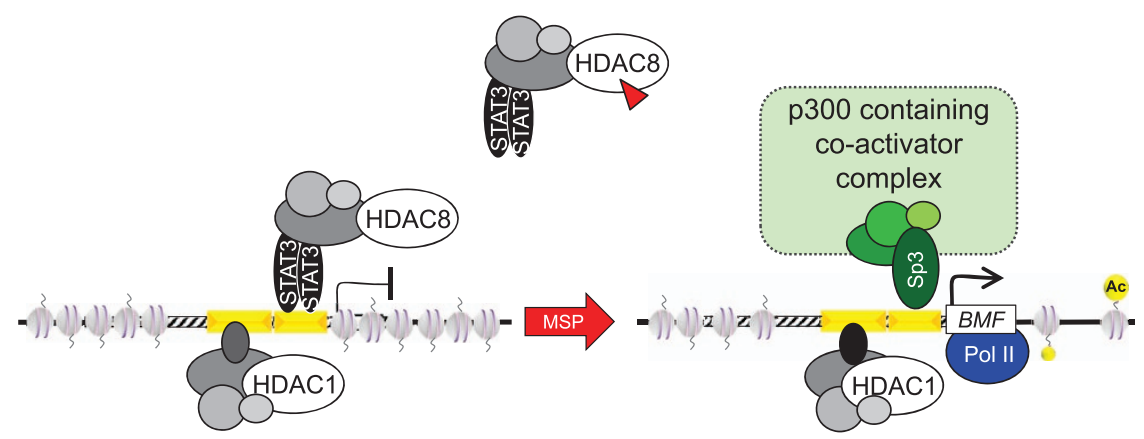

Figure 6 Model for HDAC regulation of BMF transcriptional activity. MSP inhibits HDAC8 more effectively than HDAC1 $1{ }^{10}$ which triggers the release of a corepressor complex comprising HDAC8, STAT3, and other partners. Release of the HDAC8 corepressor complex recruits p300, Sp3, and other factors that drive RNA Pol II activity

time taken to convert MSC to MSP at levels that might inhibit HDAC activity. ${ }^{10,11}$ We did not specifically examine changes in BMF expression or HDAC promoter occupancy in other cell lines shown (Supplementary Figure 4), or in colon cancer cells other than HCT116 and HT29 (Figure 2a). Such experiments might help to clarify the working model proposed here.

A notable feature of the working model for MSP (Figure 6) is the continued interaction of HDAC1 on the BMF promoter following the release of HDAC8. Time-course ChIP assays might indicate whether HDAC1 is released at later times, perhaps coinciding with the appearance of activating histone methylation marks, such as H3K4me3 (Supplementary Figure 1c). It would be interesting to assess whether, over time, other HDACs substitute at the position normally occupied by the HDAC8 complex to downregulate the target gene. This could have implications for therapeutic strategies selectively targeting HDAC8 versus other HDACs. HDAC8 alone was implicated in the pathogenesis of neuroblastoma, ${ }^{19}$ but multiple HDACs are dysregulated in most other cancers. ${ }^{17,18} \mathrm{ChIP} / \mathrm{re}-$ ChIP assays in the presence of agents such as etinostat, which preferentially target class I HDACs other than HDAC8, ${ }^{17}$ might provide insights into the roles of HDAC1, HDAC2, and HDAC3 in regulating $B M F$ expression in the absence of HDAC8. Studies with other HDAC inhibitors also might identify changes in the composition of the corepressor complex that mask specific HDAC associations, rather than necessarily triggering HDAC release from the BMF gene promoter.
In the case of vorinostat (suberoylanilide hydroxamic acid, SAHA), BMF was highly induced compared with MSP under the same conditions at $4 \mathrm{~h}$, and there was de-recruitment of both HDAC1 and HDAC8 (Supplementary Figure 6). SAHA is an established 'pan-HDAC' inhibitor, ${ }^{17,18}$ whereas MSP has 10-fold greater inhibitory activity toward HDAC8 than HDAC $1{ }^{10}$ Molecular modeling revealed that residue W141 in HDAC8 is replaced by L141 in HDAC1, and this results in less favorable docking of MSP in the active site (Supplementary Figure 7). We speculate that structural features allow MSP to preferentially target HDAC8 for release according to the working model (Figure 6), whereas panHDAC inhibitors like SAHA de-recruit both HDAC1 and HDAC8 corepressor complexes to maximally induce the $B M F$ target gene (Supplementary Figure 8).

ChIP assays also revealed that SAHA had little effect on Sp3 interactions, whereas STAT3 levels were markedly reduced on the BMF promoter (Supplementary Figures $6 \mathrm{c}$ and $d$, black versus white bars, region ' $f$ '). These findings further support the 'bystander' role of Sp3, proposed above, and the repressive actions of STAT3 on the target gene. Co-IP experiments identified a modest increase in STAT3 acetylation following MSP and SAHA treatment (Supplementary Figure 9a), suggesting a possible role for post-translational modifications to STAT3. However, in HCT116 cells expressing knock-in mutants of STAT3, a STAT3 phosphorylation mutant that interferes with DNA interactions ${ }^{23}$ consistently enhanced the basal levels of BMF, whereas a STAT3 acetylation mutant ${ }^{24}$ 
had less impact on the target gene (Supplementary Figure 9b). Interestingly, in all three cell lines, there was a comparable time-dependent increase in BMF expression that plateaued between 6 and $24 \mathrm{~h}$ after MSP treatment (Supplementary Figure 9b). We interpret these data as evidence that, beyond STAT3 post-translational modifications, HDAC8 inhibition and de-recruitment likely serves as the primary driver of $B M F$ transcriptional activity in response to MSP.

Prior work established that the silencing of HDAC8 by RNAi activated apoptosis and/or inhibited the growth of human cancer cells. ${ }^{25,26}$ No significant changes in BMF mRNA levels were detected after treatment of colon cancer cells with 5-aza-2'-deoxycytidine (Supplementary Figure 10), implying that DNA promoter methylation did not have a critical role in silencing $B M F$ transcription under the conditions reported here.

Finally, the current findings provide possible new leads for understanding the biological roles of HDAC8 in schistosomiasis, ${ }^{27}$ chronic obstructive pulmonary disease, ${ }^{28,29}$ and skull morphogenesis. ${ }^{30,31}$ Interestingly, novel mutations affecting HDAC8 and EP300 recently were linked to the development of Cornelia de Lange syndrome (CdLS), Rubinstein-Taybi syndrome, and other congenital abnormalities. ${ }^{32-35}$ Loss-of-function HDAC8 mutations in six CdLS probands increased the acetylation and loading onto chromatin of SMC3, a 'cohesin' that has a role in regulating gene expression. ${ }^{32}$ Another member of the cohesin complex, NIPBL (nipped-B-like), is commonly mutated in patients with CdLS, and the corresponding loss-of-function zebrafish embryos have increased apoptosis in developing neural tissues. ${ }^{34}$ Chemical activation of the pathway in nipblb-loss-of-function embryos rescued the adverse phenotypes and restored the physiological levels of cell death. ${ }^{34}$ This is consistent with the working model proposed here (Figure 6), in which functional HDAC8 associates with, and represses, pro-apoptotic BMF, whereas loss-offunction HDAC8 mutants would aberrantly activate apoptosis and have deleterious consequences during embryo development. On the basis of the results presented here, we speculate that dysregulation of Bmf-mediated apoptosis might have a critical role in CdLS and related syndromes.

\begin{abstract}
Materials and Methods
Cell culture. HCT116 $6^{\text {p21+/+ }}$ and HCT116 $6^{\text {p21-/- }}$ cells were provided by Dr. Bert Vogelstein (Johns Hopkins University, Baltimore, MD, USA). Dr. Zhenghe Wang (Case Western Reserve University, Cleveland, OH, USA) kindly supplied HCT116 cells expressing acetylation and phosphorylation mutants of STAT3 ${ }^{23,24}$ All other cell lines were obtained from ATCC (Manassas, VA, USA) and were cultured under the conditions described elsewhere. ${ }^{36-43}$ Chemicals were from the sources reported $^{10,11,43}$ and were added to cells at the following nominal concentrations, unless stated otherwise: MSP $(10 \mu \mathrm{M})$, MSC $(50 \mu \mathrm{M}), \operatorname{KMSB}(10 \mu \mathrm{M}), \mathrm{SM}(50 \mu \mathrm{M})$, and SAHA $(3 \mu \mathrm{M})$. In some experiments, $0,0.1,0.5$, or $2 \mathrm{mM}$ concentrations of AOAA (Sigma, St. Louis, MO, USA) were added $1 \mathrm{~h}$ before other test compounds to inhibit the transamination reaction. ${ }^{44,45}$ Other experiments used a p300 inhibitor ${ }^{46}$ (10 $\mu \mathrm{M}$ C646, Sigma), added to cells 30 min before MSP treatment, or DNA methyltransferase inhibitor 5-aza-2'-deoxycytidine, as reported. ${ }^{40}$ Cells were collected at the times indicated in the figures.
\end{abstract}

Cell viability and cell cycle analyses. MTT assays and fluorescenceactivated cell sorting analyses were conducted according to the basic protocols reported elsewhere. 47,48
Western blot. Whole-cell lysates were immunoblotted using the methodology described elsewhere. ${ }^{36,37}$ Primary antibodies were to the following acetylated (ac), methylated (me), and phosphorylated (ph) histone marks: H3K9me3, H3K4me2, H4acK12, and H4acK5,K8,K12,K16 (Upstate, Temecula, CA, USA); H3acK9,K14, H3acK9, and H3S10ph (Cell Signaling, Beverly, MA, USA); H3K27me3, H3K4me3, H3S28ph, and H3T3ph (Active Motif, Carlsbad, CA, USA); and H3acK18 (Abcam, San Francisco, CA, USA). Other primary antibodies were to histone $\mathrm{H} 3$ and histone H4 (Upstate, Cell Signaling, Active Motif); PARP, p21, Bmf, cleaved caspase-3, cleaved caspase-6, cleaved caspase-8 and cleaved caspase-9 (Cell Signaling); HDAC8 (R\&D Systems, Minneapolis, MN, USA); HDAC1 and p300 (Santa Cruz Biotechnology, Dallas, TX, USA); and $\beta$-actin (Sigma). Antibodies to p53, acetylated p53, $\alpha$-tubulin, acetylated $\alpha$-tubulin, STAT3, Sp1, and Sp3 were as reported. ${ }^{10,11,36,37,48-51}$

Knockdown and overexpression experiments. HDAC8 overexpression was achieved by transient transfection of $0.5 \mu \mathrm{g} H D A C 8$ construct or empty vector (OriGene, Rockville, MD, USA), using the protocol reported before for HDAC3 and $H_{D A C 6}{ }^{36}$ In knockdown studies, cells were transfected with Bmf siRNA (SASI_Hs01_00127817, Sigma) or negative control siRNA (Universal Negative control \#1, Sigma) using Lipofectamine RNAiMAX (Invitrogen, Carlsbad, CA, USA), according to the manufacturer's instructions. After $48 \mathrm{~h}$, cells were treated with MSP or vehicle and collected at $12 \mathrm{~h}$. RNAi also was used as reported ${ }^{48-51}$ to knockdown Sp1, Sp3, and STAT3.

Real-time RT-PCR and qPCR. Methodologies for RNA extraction, cDNA synthesis, and qPCR were reported before. ${ }^{10,40,41}$ PCR reactions were performed on a Roche Light Cycle 480 II instrument (Roche, Indianapolis, IN, USA). Primers were to the following targets: BMF, BCL2, BCLXL, BAX, BAK, BIM, APAF1, and P21WAF1. Targets were normalized to internal controls indicated in the figures.

Chromatin immunoprecipitation. After treatment with MSP or vehicle for $4 \mathrm{~h}$, cells were incubated with $1 \%$ formaldehyde in cell culture medium for $10 \mathrm{~min}$ at room temperature, and then the reaction was stopped with $0.12 \mathrm{M}$ glycine for $5 \mathrm{~min}$. DNA shearing and ChIP used the ChIP-IT Express Enzymatic (Active Motif) kit, following the recommended protocol. In the IP step, anti-histone H3, anti-H3acK9, K14 (Cell Signaling), and anti-RNA polymerase II (Active Motif) were incubated overnight at $4^{\circ} \mathrm{C}$. Additional IP experiments used antibodies to Sp1, Sp3, STAT3, HDAC8, HDAC1, and p300 (from sources listed above). After eluting pull-down DNA from magnetic beads, DNA purification used the QIAquick PCR Purification kit (Qiagen, Valencia, CA, USA). Quantification of immunoprecipitated and input (control) DNA was carried out by qPCR on a Light Cycler 480 II (Roche), with Light Cycle 480 SYBR Green I Kit master (Roche). The primer sequences are available on request.

Molecular modeling, docking, and pocket binding prediction. The 3D coordinates of the human catalytic domain of HDAC8 in the inhibited conformation and HDAC1 in the active conformation were retrieved from the Protein Data Bank (PDB $1 \mathrm{~T} 67$ and 4BKX, respectively). The human catalytic domain of HDAC1 in the inhibited conformation was built through homology modeling using PDB 1767 as a starting template (Molsoft ICM 3.7-2 d, Molsoft, San Diego, CA, USA) ${ }^{52}$ Models were energetically refined in the internal coordinate space with Molsoft ICM. ${ }^{36,53}$ Five types of interaction potential were considered: (i) van der Waals potential for a hydrogen atom probe; (ii) van der Waals potential for a heavyatom probe (generic carbon of $1.7 \AA$ radius); (iii) optimized electrostatic term; (iv) hydrophobic terms; and (v) loan pair-based potential, which reflects directional preferences in hydrogen bonding. Energy terms were based on the Merck molecular force field to account for solvation free energy and entropic contribution. Modified intermolecular terms, such as soft van der Waals, hydrogen bonding, and hydrophobic terms, were included in the analyses. Conformational sampling is based on the biased probability Monte Carlo procedure, which randomly selects a conformation in the internal coordinate space and then moves randomly to a new position independent of the previous one, according to a predefined continuous probability distribution. After each random step, full local minimization improves the efficiency of the procedure. In the ICM-VLS (Molsoft ICM) screening procedure, ligand scoring is optimized to obtain maximal separation between binders and non-binders. Ligand was assigned a score according to its fit within the protein, accounting for electrostatic, hydrophobicity, and entropy parameters. ${ }^{36,53}$ The protocol was first validated by docking trichostatin $\mathrm{A}$ into the binding site and 
reproducing the crystallographic pose in HDAC8 (PDB 1T64). Binding-pocket volumes were predicted using Pocket Finder (Molsoft ICM 3.7-d). ${ }^{54,55}$

Statistical analyses. Unless indicated otherwise data were expressed as mean \pm S.D. $(n=3)$. Paired comparisons were made using Student's $t$-test. A $P$-value $<0.05$ was considered as statistically significant, and indicated as such with an asterisk in the corresponding figures.

\section{Conflict of Interest}

The authors declare no conflict of interest.

Acknowledgements. We dedicate this work to the memory of lifelong selenophile Dr. John A. Milner. Lydia Petell performed initial work-up of the ChIP assays. Dr. Bert Vogelstein (Johns Hopkins University, Baltimore, MD) kindly supplied HCT116 ${ }^{\text {p21+/+ }}$ and HCT116 ${ }^{\text {p21-l- }}$ cells, whereas Dr. Zhenghe Wang (Case Western Reserve University, Cleveland, $\mathrm{OH}$ ) provided HCT116 cells expressing STAT3 knockin mutants. This work was supported by NIH grants CA090890, CA122959, CA90176, CA111842, P30 ES00210, P30 ES023512, and a Chancellor's Research Initiative from Texas A\&M University.

1. Campbell RM, Tummino PJ. Cancer epigenetics drug discovery and development: the challenge of hitting the mark. J Clin Invest 2014; 124: 64-69.

2. Marks PA, Wu WS. Histone deacetylase inhibitors: potential in cancer therapy. J Cell Biochem 2009; 107: 600-608.

3. Lee JH, Choy ML, Marks PA. Mechanisms of resistance to histone deacetylase inhibitors. Adv Cancer Res 2012; 116: 39-86.

4. Rajendran $\mathrm{P}$, Williams $\mathrm{DE}$, Ho E, Dashwood RH. Metabolism as a key to histone deacetylase inhibition. Crit Rev Biochem Mol Biol 2011; 46: 181-199.

5. Bishton MJ, Harrison SJ, Martin BP, McLaughlin N, James C, Josefsson EC et al. Deciphering the molecular and biological processes that mediate histone deacetylase inhibitor-induced thrombocytopenia. Blood 2011; 117: 3658-3668.

6. Shah MH, Binkley P, Chan K, Xiao J, Arbogast D, Collamore M et al. Cardiotoxicity of histone deacetylase inhibitor depsipeptide in patients with metastatic neuroendocrine tumors. Clin Cancer Res 2006; 12: 3997-4003.

7. Shao Y, Aplin AE. BH3-only protein silencing contributes to acquired resistance to PLX4720 in human melanoma. Cell Death Differ 2012; 19: 2029-2039.

8. Xargay-Torrent S, López-Guerra M, Saborit-Villarroya I, Rosich L, Campo E, Roué $\mathrm{G}$ et al. Vorinostat-induced apoptosis in mantle cell lymphoma is mediated by acetylation of proapoptotic BH3-only gene promoters. Clin Cancer Res 2011; 17: 3956-3968

9. Zhang $\mathrm{Y}$, Adachi M, Kawamura R, Imai K. Bmf is a possible mediator in histone deacetylase inhibitors FK228 and CBHA-induced apoptosis. Cell Death Differ 2006; 13: 129-140.

10. Nian H, Bisson WH, Dashwood WM, Pinto JT, Dashwood RH. $\alpha$-Keto acid metabolites of organoselenium compounds inhibit histone deacetylase activity in human colon cancer cells Carcinogenesis 2009; 30: 1416-1432.

11. Lee Jl, Nian H, Cooper AJ, Sinha R, Dai J, Bisson WH et al. $\alpha$-Keto acid metabolites of naturally occurring organoselenium compounds as inhibitors of histone deacetylase in human prostate cancer cells. Cancer Prev Res 2009; 2 683-693.

12. Pinto JT, Lee JI, Sinha R, MacEwan ME, Cooper AJ. Chemopreventive mechanisms of $\alpha$-keto acid metabolites of naturally occurring organoselenium compounds. Amino Acids 2011; 41: 29-41.

13. Somoza JR, Skene RJ, Katz BA, Mol C, Ho JD, Jennings AJ et al. Structural snapshots of human HDAC8 provides insights into the class I histone deacetylases. Structure 2004; 12 $1325-1334$.

14. Vannini A, Volpari C, Filocamo G, Casavola EC, Brunetti M, Renzoni D et at. Crystal structure of a eukaryotic zinc-dependent histone deacetylase, human HDAC8, complexed with a hydroxamic acid inhibitor. Proc Natl Acad Sci USA 2004; 101 15064-15069.

15. Vannini A, Volpari C, Gallinari P, Jones P, Mattu M, Carfí A et al. Substrate binding to histone deacetylases as shown by the crystal structure of the HDAC8-substrate complex. EMBO J 2007; 8: 879-884.

16. Dowling DP, Gantt SL, Gattis SG, Fierke CA, Christianson DW. Structural studies of human histone deacetylase 8 and its site-specific variants complexed with substrate and inhibitors. Biochemistry 2007; 47: 13554-13563.

17. Delcuve GP, Khan DH, Davie JR. Targeting class I histone deacetylases in cancer therapy Expert Opin Ther Targets 2013; 17: 29-41.

18. Mihaylova MM, Shaw RJ. Metabolic reprogramming by class I and II histone deacetylases. Trends Endocrinol Metab 2013; 24: 48-57.

19. Oehme I, Deubzer HE, Wegener D, Pickert D, Linke JP, Hero B et al. Histone deacetylase 8 in neuroblastoma tumorigenesis. Clin Cancer Res 2009; 15: 91-99.
20. Zhang Y, Sif S, DeWille J. The mouse C/EBPdelta gene promoter is regulated by STAT3 and Sp1 transcriptional activators, chromatin remodeling and c-Myc expression. J Cell Biochem 2007; 102: 1256-1270.

21. Waltregny D, Glénisson W, Tran SL, North BJ, Verdin E, Colige A et al. Histone deacetylase HDAC8 associates with smooth muscle alpha-actin and is essential for smooth muscle cell contractility. FASEB J 2005; 19: 966-968.

22. Karolczak-Bayatti M, Sweeney M, Cheng J, Edey L, Robson SC, Ulrich SM et al. Acetylation of heat shock protein 20 (Hsp20) regulates human myometrial activity. J Biol Chem 2011; 286: 34346-34355.

23. Zhang P, Zhao Y, Zhu X, Sedwick D, Zhang X, Wang Z. Cross-talk between phospho-STAT3 and PLC $\gamma$ plays a critical role in colorectal tumorigenesis. Mol Cancer Res 2011; 9: 1418-1428.

24. Lee $\mathrm{P}$, Zhang $\mathrm{P}$, Herrmann A, Yang $\mathrm{C}$, Xin $\mathrm{H}$, Wang $\mathrm{Z}$ et al. Acetylated STAT3 is crucial for methylation of tumor-suppressor gene promoters and inhibition by resveratrol results in demethylation. Proc Natl Acad Sci USA 2012; 109: 7765-7769.

25. Wu J, Du C, Lv Z, Ding C, Cheng J, Xie H et al. The up-regulation of histone deacetylase 8 promotes proliferation and inhibits apoptosis in hepatocellular carcinoma. Dig Dis Sci 2013; 58: $3545-3553$

26. Higuchi T, Nakayama T, Arao T, Nishio K, Yoshie O. SOX4 is a direct target gene of FRA-2 and induces expression of HDAC8 in adult T-cell leukemia/lymphoma. Blood 2013; 121: 3640-3649.

27. Marek M, Kannan S, Hauser AT, Moraes Mourão M, Caby S, Cura V et al. Structural basis for the inhibition of histone deacetylase 8 (HDAC8), a key epigenetic player in the blood fluke Schistosoma mansoni. PLoS Pathog 2013; 9: e1003645.

28. Andresen $E$, Günther G, Bullwinkel J, Lange $C$, Heine $H$. Increased expression of beta-defensin 1 (DEFB1) in chronic obstructive pulmonary disease. PLoS One 2011; 6 : e21898.

29. Ito K, Ito M, Elliott WM, Cosio B, Caramori G, Kon OM et al. Decreased histone deacetylase activity in chronic obstructive pulmonary disease. N Engl J Med 2005; 352 1967-1976.

30. Haberland M, Mokalled MH, Montgomery RL, Olson EN. Epigenetic control of skull morphogenesis by histone deacetylase 8. Genes Dev 2009; 23: 1625-1630.

31. Harakalova M, van den Boogaard MJ, Sinke R, van Lieshout S, van Tuil MC, Duran K et al. $\mathrm{X}$-exome sequencing identifies a HDAC8 variant in a large pedigree with X-linked intellectual disability, truncal obesity, gynaecomastia, hypogonadism and unusual face. J Med Genet 2012; 49: 539-543

32. Deardorf MA, Bando M, Nakato R, Watrin E, Itoh T, Minamino M et al. HDAC8 mutations in Cornelia de Lange syndrome affect the cohesion acetylation cycle. Nature 2012; 489: 313-317.

33. Mannini L, Cucco F, Quarantotti V, Krantz ID, Musio A. Mutation spectrum and genotype-phenotype correlation in Cornelia de Lange syndrome. Hum Mutat 2013; 34: 1589-1596.

34. Pistocchi A, Fazio G, Cereda A, Ferrari L, Bettini LR, Messina G et al. Cornelia de Lange Syndrome: NIPBL haploinsufficiency downregulates canonical Wnt pathway in zebrafish embryos and patients fibroblasts. Cell Death Dis 2013; 4: e866.

35. Woods SA, Robinson HB, Kohler LJ, Agamanolis D, Sterbenz G, Khalifa M Exome sequencing identifies a novel EP300 frameshift mutation in a patient with features that overlap Cornelia de Lange syndrome. Am J Med Genet 2014; 164: 251-258.

36. Rajendran P, Delage B, Dashwood WM, Yu TW, Wuth B, Williams DE et al. Histone deacetylase turnover and recovery in sulforaphane-treated colon cancer cells: competing actions of 14-3-3 and Pin1 in HDAC3/SMRT corepressor complex dissociation/reassembly. Mol Cancer 2011; 10: 86

37. Rajendran PR, Kidane Al, Yu TW, Dashwood WM, Bisson WH, Löhr CV et al. HDAC turnover, CtIP acetylation, and dysregulated DNA damage signaling in colon cancer cells treated with sulforaphane and related dietary isothiocyanates. Epigenetics 2013; 8: $612-623$

38. Shorey LE, Hagman AM, Williams DE, Ho E, Dashwood RH, Benninghoff AD. 3,3'-diindolylmethane induces $G_{1}$ arrest and apoptosis in human acute T-cell lymphoblastic leukemia cells. PLoS One 2012; 7: e34975.

39. Beaver LM, Yu TW, Sokolowski El, Williams DE, Dashwood RH, Ho E. 3'-Diindolylmethane, but not indole-3-carbinol, inhibits histone deacetylase activity in prostate cancer cells. Toxicol Appl Pharmacol 2012; 263: 345-351.

40. Wang R, Löhr CV, Fischer K, Dashwood WM, Greenwood JA, Ho E et al. Epigenetic inactivation of endothelin-2 and endothelin-3 in colon cancer. Int $J$ Cancer 2013; 132: 1004-1012.

41. Parasramka MA, Dashwood WM, Wang R, Saeed HH, Williams DE, Ho E et al. A role for lowabundance miRNAs in colon cancer: the miR-206/Kröppel-like factor 4 (KLF4) axis. Clin Epigenetics 2012; 4: 16

42. Xu M, Dashwood RH. Chemoprevention studies of heterocyclic amine-induced carcinogenesis. Cancer Lett 1999; 143: 179-183.

43. Myzak MC, Karplus PA, Chung FL, Dashwood RH. A novel mechanism of chemoprotection by sulforaphane: inhibition of histone deacetylase. Cancer Res 2004; 64: 5767-5774.

44. Dever T, Elfarra AA. L-Methionine-dl-sulfoxide metabolism and toxicity in freshly isolated mouse hepatocytes: gender differences and inhibition with aminooxyacetic acid. Drug Metab Dispos 2008; 36: 2252-2260. 
45. Szabo C, Coletta C, Chao C, Módis K, Szczesny B, Papapetropoulos A et al. Tumor-derived hydrogen sulfide, produced by cystathionine- $\beta$-synthase, stimulates bioenergetics, cell proliferation, and angiogenesis in colon cancer. Proc Natl Acad Sci USA 2013; 110: 12474-12479.

46. Yan G, Eller MS, Elm C, Larocca CA, Ryu B, Panova IP et al. Selective inhibition of p300 HAT blocks cell cycle progression, induces cellular senescence, and inhibits the DNA damage response in melanoma cells. J Invest Dermatol 2013; 133: 2444-2452.

47. Wang R, Dashwood WM, Nian H, Löhr CV, Fischer KA, Tsuchiya $\mathrm{N}$ et al. NADPH oxidase overexpression in human colon cancers and in rat colon tumors induced by 2-amino-1-methyl-6-phenylimidazo[4,5-b]pyridine (PhIP). Int J Cancer 2011; 128: 2581-2590.

48. Nian H, Delage B, Pinto JT, Dashwood RH. Allyl mercaptan, a garlic-derived organosulfur compound, inhibits histone deacetylase and enhances Sp3 binding on the P21WAF1 promoter. Carcinogenesis 2008; 29: 1816-1824.

49. Valin A, Ouyang J, Gill G. Transcription factor Sp3 represses expression of $\mathrm{p} 21^{\mathrm{CIP} 1}$ via inhibition of productive elongation by RNA polymerase II. Mol Cell Biol 2013; 33: 1582-1593.

50. Encarnacao PC, Ramirez VP, Zhang C, Aneskievich BJ. Sp sites contribute to basal and inducible expression of the human TNIP1 (TNF alpha-inducible protein 3-interacting protein 1) promoter. Biochem J 2013; 452: 519-529.

51. Kreuzaler PA, Staniszewska AD, Li W, Omidvar N, Kedjouar B, Turkson J et al. Stat3 controls lysosomal-mediated cell death in vivo. Nat Cell Biol 2011; 13: 303-309.
52. Katritch V, Rueda M, Abagyan R Ligand-guided receptor optimization. Methods Mol Biol 2012; 857: 189-205.

53. Neves MA, Totrov M, Abagyan R Docking and scoring with ICM: the benchmarking results and strategies for improvement. J Comput Aided Mol Des 2012; 26: 675-686.

54. Bisson WH, Koch DC, O'Donnell EF, Khalil SM, Kerkvliet NI, Tanguay RL et al. Modeling of the aryl hydrocarbon receptor (AhR) ligand binding domain and utility in virtual ligand screening to predicts new AhR ligand. J Med Chem 2009; 52: 5635-5641.

55. Abagyan R, Kufareva I. The flexible pocketome engine for structural chemogenomics. Methods Mol Biol 2009; 575: 249-279.

(c) (i) Cell Death and Disease is an open-access journal published by Nature Publishing Group. This work is licensed under a Creative Commons Attribution 4.0 International Licence. The images or other third party material in this article are included in the article's Creative Commons licence, unless indicated otherwise in the credit line; if the material is not included under the Creative Commons licence, users will need to obtain permission from the licence holder to reproduce the material. To view a copy of this licence, visit http://creativecommons.org/licenses/by/4.0

Supplementary Information accompanies this paper on Cell Death and Disease website (http://www.nature.com/cddis) 